\title{
The institutional role of the Albanian Financial Supervisor Authority
}

\author{
Dr. Arber Gjeta \\ Department of Law, \\ Faculty of Economy, \\ University of Elbasan \\ Arber.gjeta@uniel.edu.al \\ Valbona Ballkoçi \\ Department of Finance and Accounting \\ Faculty of Economy, \\ University of Elbasan \\ Valbona.ballkoç@uniel.edu.al
}

\begin{abstract}
This paper aims to examine, describe and rise critical issues on the role of the Albanian Financial Supervisor Authority within the Albanian financial system. The independence of this institution is examined under the provisions of the Law and secondary legislation as the main authority which guarantee the safeguard of the system and an effective control. The relationship of the Authority with the operators of insurance, financial market and pension funding schemes is driven by the fulfillment of its institutional role: the supervision of a new and unestablished market. The banking market, on the other side, moves from monopoly to competition and there are findings that suggest an oligopoly created in Albania. Thus, the important role of the Authority to foster competition is one of the most important, due to its obligations in the EU integration process. Its institutional role and its prerogatives are examined in order to determine if there is a complete and adequate regulation of the system.
\end{abstract}

Keywords. Financial market; supervision; Authorities; independence; institutional role; operators.

\section{L'istituzione dell'Autorità di vigilanza Finanziaria e la sua missione}

L'Autorità di Vigilanza Finanziaria è stato istituito nel 2006 dall'unificazione degli altri organismi di diritto pubblico, i quali vigilavano i singoli mercati. Queste erano: a) la Commissione dei Titoli di Credito, un organo collegiale, che vigilava il mercato e gli operatori, mediatori e consiglieri che scambiano titoli di credito, etc.; b) l'Autorità di Vigilanza delle Assicurazioni che operava nel campo assicurativo; c) l'Ispettorato delle Istituti Pensioni Supplementari.

La nascita di un organo indipendente era una necessità vista lo sviluppo del mercato, ma non solo. Era una necessità anche per gli impegni presi nell'ottica d'integrazione con i mercati europei, che sono parte importante di un lungo processo verso la definitiva adesione nell'Unione Europea. Un unico organismo indipendente, quindi, che vigila le diverse parti del sistema finanziario. Scelta che si uniforma con gli ordinamenti più sviluppati e con una lunga esperienza nel campo della vigilanza dei mercati. Tale scelta fu dettata dalla sempre più spesse interferenza degli operatori in mercati diversi.

La diversificazione dell'offerta di strumenti finanziari da parte degli intermediari verso il pubblico rende necessario una simile scelta. Infatti, sempre di più, le diverse categorie degli operatori si trovano a competere negli stessi mercati.

Si sono elaborati e applicati negli anni diversi modelli di vigilanza. Si è passati dalla regolamentazione e vigilanza per soggetti (istituzionale) a quella per finalità (per funzioni) fino ad arrivare al modello dell'agenzia con funzioni di regolatore 
unico ${ }^{1}$. Le autorità di vigilanza in generale, sia del settore bancario sia degli altri mercati, stano tentando di modificare lo stile della vigilanza. Oggi si sta ponendo l'attenzione sul collegamento tra vigilanza e norme di corporate governance (Vella, 2008, p. 171). In un'ottica di vigilanza attuata tramite la validazione preventiva di codici di autodisciplina è importante che si mantenga il giusto rapporto tra vigilanza pubblica rispettosa dell'autonomia imprenditoriale e queste forme di selfregulation (Vella, 2008, p. 172).

La legge 9572 del 2006, che istituisce l'AVF, lo definisce come "una persona giuridica di diritto pubblico, indipendente, con sede a Tirana, specializzato per la regolamentazione e la vigilanza dell'attività dei soggetti ..."2. L'AVF è nata con il preciso scopo di vigilare tutti i mercati dei capitali in Albania che non appartengono al settore bancario, quindi una divisione per soggetti vigilati. II campo della sua attività è il regolamento e la vigilanza sui mercati dei titoli di credito, quello delle assicurazioni e delle riassicurazioni e alle operazioni a esse connesse, il mercato delle pensioni supplementari offerte da soggetti privati e di tutte le altre attività finanziarie non bancarie.

La teoria dei mercati che si regolano da se è stata da tempo superata. Già Adam Smith nella sua Wealth of Nations scriveva che "è difficile che delle persone dello stesso mestiere si incontrino, anche per festa o per divertirsi, senza che la conversazione finisca in una cospirazione contro lo Stato o in qualche espediente per sollevare i prezzi" (Smith, p. 155).

In un mercato cosi giovane, questo è un obiettivo importante da raggiungere, specialmente in questi tempi di crisi mondiale dei mercati e della finanza in cui le autorità regolamentari stanno cercare di frenare gli effetti della crisi e di darsi delle regole per il futuro immediato per una vigilanza più effettiva in ottica internazionale. Gli organismi internazionali, quindi, devono armonizzare le regole della vigilanza ai principi generalmente condivisi, come per esempio i principi IOSCO${ }^{3}$.

Se i mercati vanno sempre di più integrandosi e unificarsi, anche le autorità di vigilanza non devono rimanere indietro ma collaborare in maniera effettiva tra di loro. Si fa fatica oggi a vigilare i mercati e gli strumenti finanziari in essi negoziati. Le innovazioni finanziarie hanno bisogno di un aggiornamento continuo dei modelli di vigilanza. Dobbiamo dire allora che una tale Autorità con ampi poteri è necessaria in ogni sistema finanziario. Si può dire che e impensabile parlare di mercato senza un organismo indipendente che lo vigila adeguatamente.

In più, la legge albanese gli riconosce anche il preciso dovere programmatico di promuovere la creazione di un mercato sano, stabile, trasparente e affidabile ${ }^{4}$. Questo è uno dei suoi principali compiti insieme alla vigilanza e alla regolamentazione del mercato. Compito che la stessa Autorità definisce come missione insieme alla protezione dei consumatori e degli investitori che in questo mercato opereranno (AMF, 2007, p. 2). In un paese dove le azioni si scambiano ancora al mercato informale e le società registrate presso il Centro di Registrazione delle Azioni sono solo cinquanta, dove nell'unica borsa esistente si comprano e si vendono solo Buoni del Tesoro (Gazeta Standard, 2008) questo obiettivo diventa primario e di fondamentale importanza. Dobbiamo auspicare allora di vedere un'Autorità molto attiva anche nella direzione della promozione di questi mercati e renderlo appetibile per i investitori esteri. In questo momento nei mercati albanesi si sono inseriti a un buon livello gli investitori stranieri, specialmente nel campo delle assicurazioni 5 .

II lavoro dell'Autorità deve consistere nel vivacizzare il resto del mercato. Compito che può essere raggiunto tramite una completa e dettagliata informazione verso il pubblico e verso i possibili investitori esteri. Per raggiungere questi obiettivi e per adempiere la sua missione, la strada maestra è la trasparenza delle politiche seguite, delle decisioni prese e della prassi cui si deve conformare l'Autorità. Attività, queste che vanno sempre motivate analiticamente. Infatti, una tale disposizione è prevista nella legge sull'Autorità. L'articolo 11 sotto la rubrica "La trasparenza nell'attività dell'Autorità" prevede l'obbligo per l'Autorità di emanare il regolamento sulle procedure mediante le quale sarà messo a disposizione del pubblico l'informazione elaborata dall'AVF.

\footnotetext{
1 II caso inglese dove nel 1998 si istitui la Financial Services Authority che è responsabile sulla vigilanza di tutti i mercati.

${ }^{2}$ Art. 3 della I. 9572 del 03.07.2006 "Sull'Autorità della Vigilanza Finanziaria"

${ }^{3}$ Per dare un esempio si veda un parere della Banca Centrale Europea verso una proposta di direttiva in ambito dei mercati finanziari, in specifico sul prospetto da pubblicare per l'offerta al pubblico o nella negoziazione di valori mobiliari che citiamo "8. ... la BCE accoglie con favore l'obiettivo di allineare la legislazione europea sui valori mobiliari ai principi elaborati dalla IOSCO. L'adozione di principi accettati a livello internazionale, come quelli promulgati dalla IOSCO, dovrebbe far convergere i sistemi finanziari internazionali verso un regime delle informazioni relative ai valori mobiliari meglio armonizzato..." GUCE C344/4 del 06.12.2001

${ }^{4}$ Art. 12 della I. 9572 del 03.07.2006 "Sull'Autorità della Vigilanza Finanziaria"

${ }^{5} \mathrm{Nel}$ campo delle assicurazioni nel 2007 si conta un capitale estero pari al $35 \%$ del capitale investito nelle società di assicurazione (AMF, 2007, p. 14).
} 
L'AVF fa parte dell'ordinamento albanese ed si può sostenere che almeno formalmente completa il quadro normativo e regolamentare dei mercati finanziari. Essa possiede le qualità necessarie di poter dare uno stimolo al mercato e di ritagliarsi cosi un ruolo effettivo ed efficiente in esso. Resta da vedere se questo succederà o se il mercato albanese continuerà anche in questi anni a svilupparsi in mercati sommersi o peggio illegali. Dati alla mano, adesso è ancora presto per dare un giudizio sull'effettivo impatto dell'Autorità nel mercato albanese.

\section{Struttura, compiti e organizzazione dell'Autorità}

Come ogni organismo che deve funzionare correttamente anche l'Autorità ha bisogno di una struttura efficiente. Non si possono conferire delle competenze a un ente se egli non gode della possibilità di adempiere ad esse. La legge d'istituzione dell'Autorità di Vigilanza Finanziaria prevede una struttura abbastanza complessa dell'ente ma con compiti importanti riservati al suo organo apicale che è la Commissione. Divisa in quattro dipartimenti, di cui i due sono più importanti ${ }^{1}$ e sono diretti dai due vice presidenti, essa ha un personale di 45 impiegati. A questi dipartimenti fanno capo diversi uffici, ciascuna con compiti precisi².

Essendo costituito solo di recente non possiamo escludere che nell'immediato futuro la sua struttura organizzativa possa subire dei cambiamenti. Cambiamenti che possano rispecchiare l'esperienza di questi primi due anni e le esigenze di organizzazione. Perché è vero che la normativa che regola l'Autorità e frutto di un lavoro coordinato a livello internazionale con i vari partner come $\mathrm{FMI}_{0} \mathrm{BM}^{3}$, ma e anche vero che per svolgere con efficienza i propri compiti l'Autorità deve adattarsi alle esigenze del mercato e del suo sviluppo. Non si può pretendere di applicare il miglior modello legale se questo non si adatta alle esigenze del mercato e non rispecchia le peculiarità dell'ordinamento.

L'AVF, abbiamo detto, è un'autorità giovane, la quale è stata istituita solo di recente con la legge 9572. In questa legge l'Autorità trova il fondamento delle sue funzioni. Enuncia gli obiettivi dell'Autorità l'art.12, nel quale si sancisce che "nel suo operato l'Autorità s'indirizza da questi obiettivi: 1. tutelare gli investitori e le persone assicurate. 2. promuovere la stabilità, trasparenza e affidabilità dei mercati finanziari non bancari. 3. assicurare il rispetto della legge". Nel perseguire questi obiettivi l'Autorità svolge una vasta attività che consiste sia nella vigilanza regolamentare, sia in quella ispettiva e informativa. L'art. 13 individua come mezzi necessari per raggiungere gli obiettivi la possibilità di emanare atti regolamentari previsti dalla legge, circolari e materiali informativi vari. Nel suo secondo punto nella legge si usa la locuzione "vigilanza statale". Secondo me bisogna intenderla come vigilanza pubblica perché l'Autorità non è più parte dell'apparato statale $4 \mathrm{e}$ non e non svolge i propri doveri per assicurare una vigilanza statale al mercato ma per garantire come arbitro il suo normale funzionamento. La verità è che a quest'organo sono delegati poteri propri di diritto amministrativo ma l'Autorità funziona come un organismo indipendente e autonomo.

La vigilanza, per essere effettiva, non si sente il bisogno che sia pubblica. Vediamo, per esempio, il caso inglese, dove la vigilanza è stata affidata a una società privata, la FSA, con una chiara funzione pubblica che è la vigilanza su tutti i mercati di capitale. Cosi, dobbiamo intendere vigilanza pubblica invece che statale, perché la legge possa trasmetterci i principi internazionali generalmente condivisi in materia di vigilanza.

Attualmente, il lavoro dell'Autorità è concentrato sulla emanazione dei vari regolamenti che completeranno il quadro legale e operazionale nel mercato (AMF, 2008, p. 55). Tra i regolamenti più importanti approvati possiamo menzionare: Reg. 4 del 08.02.2007 "Sui limiti per l'investimento del capitale delle società di assicurazione", reg. 120 del 02.10.2008 "Sull'autorizzazione e vigilanza delle borse valori", reg. 115 del 11.09.2008 "Sul contenuto e sulla tenuta del libro dei ordini e del libro delle transazioni", reg. 11 del 22.02.2008 "Sulla vigilanza e il controllo dei istituti privati di pensioni supplementari", etc.

La vigilanza regolamentare è svolta dalla Commissione, la quale emana i regolamenti, rilascia le autorizzazioni,

\footnotetext{
1 Sono il Dipartimento della Vigilanza e Regolamentazione e il Dipartimento Autorizzazioni e Monnitoraggio. Sempre sotto la direzione dei vicepresidenti poi sono anche gli altri due dipartimenti che sono quello delle Questioni Giuridiche e il Dipartimento della Statistica e Ricerca.

2 Gli uffici sono costituiti in base alla legge. Fra i più importanti possiamo menzionare quello della Rapporti e Analisi, Ispezione, Amministrazione del rischio, Autorizzazioni e Monitoraggio, etc. (AMF, 2008, p. 52)

3 La Banca Mondiale è uno dei principali partner dell'Autorità dalla creazione. Collaborazione che si estende in diverzi campi: miglioramento quadro normativo, metodologie di vigilanza, etc. Questi sono progetti hanno portato un'adatamento più veloce del personale ai compiti da svolgere e un migliore funzionamento dell'Autorità. (AMF, 2008, p. 37)

${ }^{4}$ Come lo erano le sue precedenti le quali vigilavano nei diversi settori. Vedi supra p. 13
} 
etc. mentre la vigilanza sul campo è svolta dagli ispettori dell'Autorità su ordine del Presidente della Commissione o di chi ne fa le sue funzioni. L'ispezione è disposta dalla Commissione e firmata dal Presidente ma è il personale che attua l'ordine ispettivo secondo le procedure previste dalla legge. L'art. 19 dispone che "gli impiegati ordinati, in ..., ispezionano per: 1. Vedere se sono stati rispettati le diposizioni legali e gli atti subordinati in vigore che regolano l'attività dei mercati finanziari non bancari; 2. Prevenire e identificare le violazioni ..."

Mentre per quanto riguarda la vigilanza informativa possiamo dire che siamo abbastanza indietro nell'attuare i compiti di invio dati verso l'Autorità e nel vigilare costantemente gli operatori tramite il loro dati inviati. Comunque, sono vari gli obblighi informativi che sorgono in capo ai soggetti vigilati. Per esempio art. 15 della I. 9879 del 21.02.2008 "Sui titoli" dispone che "emittente e il registratore informano l'Autorità, in ogni caso, quando durante un periodo di 12 mesi, i diritti sulle azioni con diritto di voto e che costituiscono almeno il $5 \%$ del capitale societario sono stati trasferiti più di una volta

.... Possiamo menzionare anche gli obblighi informativi che gli emittenti dei titoli hanno nei confronti dell'Autorità sia prima del collocamento nel mercato, con la pubblicazione del prospetto informativo, sia dopo, quando sono obbligati a informare l'Autorità sulla percentuale dei titoli sottoscritti. Vediamo allora che l'obbligo informativo è previsto in numerosi casi che riguardano sia l'intermediario, sia le operazioni che egli compie. La sanzione per il venir meno agli obblighi informativi è drastica. Il soggetto autorizzato perde il diritto di negoziare qualsiasi titolo nel mercato1.

Infine, la vigilanza ispettiva è il completamento dei poteri dati all'Autorità. Essa è esercitata sia sul luogo (on site),tramite le ispezioni disposte dalla Commissione, sia a distanza (off site), tramite il controllo dei documenti inviati dai soggetti vigilati. La vigilanza effettuata secondo questo schema dovrebbe essere effettiva ma adesso non possiamo esprimere un giudizio sull'operato dell'Autorità visto che in questi due anni si e lavorato più per formare l'AVF che per vigilare il mercato. I due rapporti annuali (AMF, 2007; AMF 2008) pubblicati illustrano un lavoro diretto all'individuazione dei compiti che l'AVF deve svolgere in pratica per raggiungere i propri obiettivi e del consolidamento della sua autorità verso gli operatori. Accanto alle statistiche di vigilanza esiste anche una cospicua parte informativa sui compiti dell'Autorità nell'immediato futuro e sugli obiettivi che essa si propone di raggiungere.

\subsection{La Commissione come il più alto organo decisionale}

L'organo deliberativo dell'Autorità di Vigilanza è la Commissione. Come la maggior parte delle autorità, anche quella albanese ha scelto come organo decisionale un organo collegiale, composto di 7 membri composta dal Presidente, due Vicepresidenti e 4 altri membri i quali vengono approvati direttamente dal organo legislativo.

Le proposte sottoposte al vaglio parlamentare sono fatte da diversi soggetti. Cinque dei candidati sono proposti al Parlamento da parte del Consiglio dei Ministri mentre la Commissione Parlamentare dell'Economia e Finanza e la Commissione delle Questioni Legali, Pubblica Amministrazione e i Diritti dell'Uomo propongono ciascuna i loro candidati. A loro volta, delle cinque candidature che il Consiglio dei Ministri può proporre, solo due sono esclusivamente di propria scelta. Gli altri tre nomi sono sottoposti al Consiglio dei Ministri rispettivamente dal Ministro delle Finanze, Consiglio di Vigilanza della Banca D'Albania e dal Consiglio Nazionale Contabilità. Si può dire, quindi, che il processo per la nomina dei membri della Commissione è un processo lungo e complesso, il quale rende partecipi quasi tutti i soggetti pubblici che operano nel mercato. Si deve dire, però, che l'approvazione definitiva passa anche qui, come per la Banca D'Albania, per mano del Parlamento. Ė un processo politico dove le negoziazioni sono molto estenuanti e la conclusione è una risultante di tutti gli attori e interessi pubblici in campo. Si vede chiaramente che il legislatore si è riservato la facoltà di nominare i membri della Commissione. Un indice d'indipendenza dell'Autorità che ancora una volta ci mostra lo stretto legame Autorità-legislatore. Legame diretto che più chiaramente si vede nell'obbligo dell'Autorità di rendere conto del suo operato solo al Parlamento almeno una volta all'anno secondo l'art.28. Entro aprile dell'anno successivo sono presentati al Parlamento 3 documenti contemporaneamente: 1 . un conto sullo stato finanziario annuale; 2 . II rapporto sulla chiusura del bilancio; 3. II rapporto annuale sulla sua attività. II rapporto annuale deve racchiudere ogni informazione richiesta dal legislatore e obbligatoriamente anche i dati sull'adeguatezza della struttura legale del mercato regolamentato, tutte le autorizzazioni approvate, quelle negate e gli altri atti amministrativi, le decisioni dell'Autorità contro cui e stato presentato ricorso e dettagliatamente quelle dove il ricorso è stato accettato e le iscrizioni ai registri dei operatori. In più, il rapporto deve contenere il risultato delle ispezioni effettuate, le politiche d'informazione verso il pubblico e quelle di collaborazione interna e internazionale, il modo in cui sono state effettuate le scelte del personale e ogni altra informazione che la

${ }^{1}$ Art. 57, pt. 1 , nr. 11 della I. 9879 del 21.02.2008 "Sui titoli" 
Commissione pensa di includerli.

Una volta nominati dal Parlamento loro restano in carica per 5 anni con la possibilità di un altro mandato solamente. Durante il loro mandato il Presidente e i suoi vice non possono svolgere nessun altro incarico salvo quelli inerenti ai loro impegni accademici, mentre gli altri 4 membri possono lavorare anche a tempo pieno. L'unico vincolo imposto a loro consiste nella preclusione di essere membri di più di un consiglio direttivo di un'altra autorità di vigilanza. I candidati per la Commissione devono godere di certi requisiti per poter essere scelti. Loro devono essere cittadini albanesi laureati in scienze economiche, giuridiche o lauree affini a esse. Devono possedere, inoltre, "un'esperienza di almeno tre anni in uno dei seguenti campi: finanze e contabilità, amministrazione società, consulenza legale verso le società, legal drafting, assicurazioni e pensioni o attività universitaria a queste connesse ..."1. In più devono godere di certi requisiti morali, non essere mai stati condannati e non possono avere cariche dirigenziali in partiti politici. L'art. 5 menziona anche il conflitto di interessi come uno delle condizioni di ineleggibilità e decadenza.

I membri della Commissione e il personale si devono conformare durante lo svolgimento del loro lavoro al codice etico e gli parametri professionali approvati dalla Commissione secondo la proposta fatta dal Presidente. Le sanzioni vengono irrogate dalla Commissione e vano fino all'esonero dalla carica o licenziamento dal lavoro. Per ogni condizione sopravenuta il membro deve riferire al Parlamento. Si vede cosi, che si istaura anche un rapporto diretto del Parlamento con ogni membro della Commissione e non soltanto con l'Autorità in generale.

II conflitto d'interessi è regolato dall'art. 22 della I. 9572 del 03.07.2006, il quale dispone che i membri della Commissione e le persone che lavorano per l'Autorità devono rendere pubblica la loro dichiarazione dei redditi. I membri della Commissione non partecipano alle riunioni nelle quali si discutono casi dove loro hanno un interesse diretto o indiretto e il personale non deve partecipare nelle ispezioni verso i soggetti vigilati se esiste una questione nella quale lui ha un interesse diretto o indiretto. La situazione del conflitto d'interessi va dichiarata per iscritto dal soggetto interessato, il quale si deve astenere dal esercitare i suoi compiti nel caso concreto pena l'immediato esonero dalla carica. Si vede che la sanzione è drastica. La lettera della legge è chiara. La figura del membro o del personale dell'Autorità deve essere pulita oltre ogni dubbio. Mentre poco chiara sembra nel disporre che "la misura dell'esonero o dell'allontanamento dalla carica non incide nelle decisioni prese fino a quel momento"2. Quindi l'esonero del membro non invalida le decisioni nelle quali egli ha votato come parte della Commissione. Normalmente questa è una norma che da stabilità e certezza agli operatori dei mercati che senz'altro hanno un interesse in tale scelta. Infatti, sarebbe impensabile che un operatore si vedesse revocata la propria licenza, la propria richiesta per operare fuori dai confini o altro solo perché in quella seduta ha preso parte un membro della Commissione non legittimato a farlo.

Cosa si può dire della decisione viziata dal suo interesse personale nel caso? Come dobbiamo capire questa norma riguardo alla questione posta? La decisione sopravvive per salvaguardare l'affidamento dei terzi o è annullata dalla Commissione?

In attesa che a questi quesiti sia data risposta dalla futura prassi della nuova autorità noi auspichiamo un veloce intervento anche di natura regolamentare dell'AVF sul quesito allineandosi e applicando i principi del diritto comune sull'annullabilità e sulla nullità prendendo spunto magari anche dalla soluzione applicata dalla legge sulla Banca D'Albania.

Fin adesso abbiamo visto cosa fa la Commissione, i suoi obiettivi e come vengono scelti i soggetti che lo compongono mentre nel prossimo paragrafo vediamo come lo fa e quali sono i suoi poteri.

(segue) 2.2- Le competenze della Commissione

Abbiamo visto che la Commissione è composta dal Presidente, due Vicepresidenti e 4 membri, i quali non hanno compiti esecutivi. Si deve dire che non solo il Presidente della Commissione ma anche gli altri due membri con funzioni esecutive, i Vicepresidenti, nel momento poiché sono proposti, sono determinate le loro cariche e i dipartimenti che andranno a dirigere.

Una volta formatosi la Commissione, essa comincia a svolgere le sue funzioni come organo collegiale. Le sue riunioni si convocano su richiesta di ciascuno dei membri e sono presiedute dal Presidente o da uno dei suoi Vicepresidenti

\footnotetext{
${ }^{1}$ Art.5, pt. 3 della 1.9572 del 03.07.2006

2 Art. 22, pt. 5 della I. 9572 del 03.07.2006 "Sull'Autorità della Vigilanza Finanziaria"
} 
delegati. Le decisioni si prendono a maggioranza dei membri della Commissione. Non è ammessa l'astensione dal voto. competenze:

Le competenze della Commissione sono elencate nell'art. 14 della legge sull'Autorità. Essa ha queste

1. Redige le regole che descrivono e illustrano lo scopo del lavoro di ogni dipartimento;

2. Emana i principi base che la sua attività deve conformarsi;

3. Emana i regolamenti e le direttive previste dalla legge; secondo grado;

4. Distribuisce materiale informativo e circolari sulla corretta interpretazione e applicazione delle leggi e gli atti di

5. Approva il format delle autorizzazioni che si rilasciano per l'esercizio di una delle attività vigilate dall'Autorità;

6. Rilascia, rifiuta, sospende o ritira le autorizzazioni per l'esercizio dell'attività in uno dei mercati vigilati;

L'Autorità svolge questo compito vigilando sistematicamente sull'applicazione delle due decisioni, rilasciando le autorizzazioni per gli operatori che operano in tutti i tre i mercati, approvando i prospetti informativi delle operazioni sul mercato.

In più, è lei che, come il più alto organo di controllo, che deve approvare ogni trasferimento di proprietà delle società vigilate, ogni loro cambiamento dello statuto, cessione di attività, scissione o fusione, vigila l'applicazione delle decisioni prese sulle offerte pubbliche sui titoli, etc. ${ }^{1}$

7. Limita o interrompe l'attività delle società di assicurazione o riassicurazione quando gli investimenti sono in contrasto con i rapporti regolatori stabiliti con regolamento dell'Autorità ${ }^{2}$ o quando ce un rischio per la posizione finanziaria della società;

La domanda che si pone su questa norma è se il rischio per la posizione finanziaria della società sia una valutazione d'opportunità fatta dall'Autorità secondo la sua discrezionalità oppure se, come pare opportuno, l'Autorità si debba uniformare alle esperienze e ai principi che ispirano le altre sue simili nel vigilare i mercati ma non solo.

L'Autorità si deve conformare, nelle sue decisioni, anche agli impegni presi in sede di associazione all'International Association of Insurance Supervisors (IAIS), nella quale è associata. Essa cosi condivide e applica nella sua attività regolamentare i principi generali (IAIS, 2002) approvati in questa sede. Prendendo spunto da queste riflessioni vediamo la percentuale del rischio che effettivamente una società di assicurazione può assumere per legge. In Albania nessuna società di assicurazione non può accollarsi un rischio maggiore del $10 \%$ del suo capitale netto. Allineandosi alla normativa europea, con la finalità di crescere la stabilita finanziaria delle società delle assicurazioni (Minxhozi, 2009, $p$. 41), nel 2008 si è stabilito il minimo del capitale nella somma di 3 milioni di euro.

8. Entro un mese dalla richiesta, deve prendere visione delle condizioni del contrato che una società vuole lanciare nel mercato;

9. Approva le situazioni finanziarie delle società d'assicurazione e riassicurazione; gli operatori;

10. Approva e distribuisce degli standard di contabilità e i format di redazione dei dati finanziari obbligatori per

11. Approva una lista dei revisori (esperti contabili) che possono essere scelti dalle società che operano nei mercati regolamentati;

12. Decide, nei casi previsti dalla legge, per l'apertura del fallimento dei soggetti vigilati e quanto è previsto dalla legge nomina il curatore fallimentare (liquidatore);

13. Stabilisce sui compensi e sugli stipendi percepiti dal personale;

14. Decide in merito agli accordi di collaborazione internazionale e sulla possibilità di scambiare informazioni su

\footnotetext{
${ }^{1}$ Art. 14, pt. 1, n. 6, a)-n) della I. 9572 dello 03.07.2006 "Sull'Autorità della Vigilanza Finanziaria"

2 Sono stabilite con regolamento i limiti di partecipazione delle società di assicurazione nelle società bancarie (solo per il $25 \%$ del loro capitale), i limiti di investimento nei mercati esteri di capitale (investimenti solo in titoli di stato dei stati UE o dell'America del Nord dopo aver preso il nulla-osta da parte dell'AVF), i limiti di partecipazione nelle persone giuridiche non bancarie (i limiti sono di $10 \%$ del capitale della società d'assicurazione), etc. Reg. Nr. 4 del 08.02.2007 "Per kufijte e investimit te kapitalit te shoqerive te sigurimit"
} 
basi di reciprocità con le autorità interne o estere;

15. Dibatte periodicamente sui rapporti dell'operato dei due dipartimenti;

16. Approva il bilancio di previsione e il rapporto annuale dell'Autorità;

17. Approva il rapporto sull'industria (economia reale) e il collegamento con i mercati regolamentati e le sue prospettive;

18. Approva, nei regolamenti sulle procedure interne, gli termini nei quali l'Autorità provvede sulle questioni a lei sottoposte;

19. Approva i manuali dell'ispezione secondo i quali saranno attuati i controlli verso gli operatori; non bancario;

20. Determina le politiche di vigilanza, del controllo dei soggetti vigilati e dello sviluppo del mercato finanziario

21. Decide sulle consulenze esterne e sul compenso dei consulenti;

22. Approva delle regole sul reclutamento o licenziamento dei dipendenti dell'Autorità;

23. Altre competenze previste da questa legge $o$ da altre leggi sulla materia.

Vediamo allora in quest'art. 14 una elencazione abbastanza esaustiva delle competenze, ma comunque non tassativa. II punto 23 non esclude competenze della Commissione previste in altre leggi. Sarebbe stata invece auspicabile una norma di chiusura che stabilisse la funzionalità delle competenze al raggiungimento del fine e degli obiettivi dell'Autorità. Gli compiti cosi elencati della Commissione, possono essere raggruppati in tre diversi gruppi. Ci sono compiti primari per il raggiungimento degli obiettivi, compiti a questi secondari o strumentali e compiti di servizio. L'apparato dell'Autorità deve essere sufficiente per il momento a svolgere i compiti previsti nella legge. II problema, se mai, può crearsi nel futuro con lo sviluppo dei titoli negoziati e la crescita del numero dei operatori che operano nei mercati. II lavoro della Commissione si deve indirizzare in quei compiti che sono primari al raggiungimento degli obiettivi dell'Autorità ma senza tralasciare quelli a essi strumentali.

\section{2.a. Le competenze dei membri della Commissione come singoli. II ruolo del Presidente}

I singoli membri accanto alle loro funzioni come parte della Commissione hanno anche dei poteri propri che esercitano sia al interno dell'Autorità sia verso i soggetti vigilati. Esercitando questi poteri loro si tengono informati adempiendo meglio anche il loro compito deliberativo.

II Presidente della Commissione, il quale nel collegio deliberativo ha un ruolo parificato agli altri membri, vediamo che ha una serie di competenze importanti elencate nell'art.15. Egli è il direttore esecutivo generale e amministratore principale dell'Autorità. Ė sotto la sua competenza tutta l'organizzazione amministrativa dell'Autorità salvo quella devoluta espressamente alla competenza dei vicepresidenti. II Presidente è anche il rappresentante dell'Autorità nelle sue relazioni verso l'esterno, convoca e dirige le riunioni della Commissione, indirizza il lavoro dei vicepresidenti, dirige il lavoro della stesura e presenta davanti alla Commissione il bilancio di previsione, può sollevare comitati consultivi per l'esercizio delle sue funzioni, etc. In più, e sempre il Presidente che sottoscrive le denunce penali per le azioni o omissioni dei dipendenti che costituiscono reato. È egli che deve sottoscrivere la notizia di reato da spedire alla procura. Normalmente poi, è la Procura che si deve attivare per esercitare l'azione penale. I compiti devoluti al Presidente sono i naturali compiti di un organo dirigente. Per esercitare i suoi poteri esso dispone del suo Gabinetto e della possibilità di poter commissionare consulenze esterne. Importante è soffermarsi nel dovere che la legge gli riconosce di tenere i rapporti istituzionali di collaborazione con la Banca D'Albania e le altre autorità albanesi. Collaborazione che va dalla reciproca informazione tra le istituzioni fino all'ispezione congiunta verso i soggetti vigilati. L'art. 15, infatti, prevede che il Presidente "9. scambia informazioni con la Banca D'Albania, con altre istituzioni e agenzie governative, con autorità e amministrazioni locali, con enti non-profit, le quali esercitano attività legate con i mercati finanziari non bancari, vigilate dall'Autorità e con i soggetti vigilati" e che "11. chiede alla Banca D'Albania di intraprendere ispezioni con oggetto definito nelle banche di secondo livello, in conformità anche con la legge "Sulle banche" e di informarla dei risultati del controllo".

In più, collaborando con la Banca D'Albania e con ordine d'ispezione congiunta chiede l'ispezione delle banche di secondo livello "che sono depositarie dei soggetti autorizzati dall'Autorità di esercitare l'attività in uno dei mercati 
finanziari non bancari, vigilate dall'Autorità'".

Vediamo cosi una possibilità di collaborazione effettiva tra le due autorità di vigilanza. Questo porterà a una copertura totale del mercato e a una vigilanza efficiente. In questo momento, tale collaborazione, possiamo affermare che esiste. Nell'affermare questo fatto dobbiamo menzionare anche il ruolo preminente che la Banca D'Albania gode de facto.

In più, esiste anche una collaborazione al rango di vertice tra i due soggetti e anche il Governo nel Gruppo di Consiglio sulla Stabilità Finanziaria. Questa collaborazione si manifesta per di più nell'informarsi a vicenda sulle questioni attinenti vigilanza dei rispettivi mercati. Le competenze dei vicepresidenti, come abbiamo già visto in precedenza ${ }^{2}$, sono quelle di dirigere i dipartimenti dell'Autorità e per quelle sono pienamente responsabili mentre le altre competenze amministrative vengono definite dalla Commissione stessa.Accanto agli amministratori, abbiamo detto che ci sono anche i 4 membri senza funzioni esecutive. Loro possono fungere da comitato di controllo per specifiche questioni sottoposte alla Commissione legate a violazioni durante l'attività dell'Autorità. Uno di essi, nella funzione del relatore, stende, infine, una relazione per la Commissione con eventuali raccomandazioni per rimuovere la violazione. In un secondo momento, se risulta opportuno, è il Parlamento a decidere per il auditing esterno sull'operato dell'Autorità ${ }^{3}$. Si nota allora che i membri della Commissione non agiscono solo come parte di essa ma hanno anche dei compiti propri all'interno dell'Autorità.

\section{II Gruppo di Consiglio sulla Stabilità Finanziaria (GCSF)}

Nella legge istitutiva dell'Autorità di Vigilanza Finanziaria si prevede anche la costituzione del GCSF che è un'unità di consulenza.

I membri del Gruppo sono: il Ministro delle Finanze; il Presidente della Commissione dell'Autorità di Vigilanza Finanziaria; il Governatore della Banca D'Albania. Vediamo allora che quest'organismo è un superconsiglio dove si discutono i problemi più importanti del sistema finanziario in generale.

La collaborazione tra i vertici degli attori principali del campo finanziario porta a un migliore coordinamento nell'adempimento dei loro compiti. La sua funzione è di assistere nello sviluppo delle politiche congiunte, collaborazione e coordinamento delle decisioni dei membri di questo consiglio nel campo dell'ispezione dei soggetti vigilati nei diversi mercati. In più, assiste nel miglioramento e l'aumento dell'efficacia dei controlli sugli operatori nei mercati finanziari. II Gruppo ha come obiettivo anche il miglioramento del quadro legale per la vigilanza. Nelle sue riunioni, su invito del Gruppo, possono partecipare anche il Capo della Commissione Parlamentare dell'Economia e delle Finanze o altre persone.

In pratica, dalle prime osservazioni, emerge che questa collaborazione si è vista in maniera costante nel campo dell'attività regolamentare dove, nel 2008 per esempio si è sviluppato il progetto di legge "Sull'assicurazione obbligatoria nel settore del trasporto" (AMF, 2008, p. 35), redatto insieme al Ministero delle Finanze oppure ai diversi regolamenti emanati con l'aiuto della Banca D'Albania. Fino adesso la collaborazione tra queste autorità e governo è stata soddisfacente e ha portato a una leale interazione tra i soggetti. Tutto questo ha portato una copertura notevole del mercato, sia finanziario, che bancario. Resta da vedere se nel futuro, quando sarà consolidata la posizione dell'Autorità come istituzione vigilante, questo consiglio continuerà a svolgere efficacemente i propri compiti o se ci sarà un allontanamento tra le istituzioni.

Fino adesso questo quadro complessivo che abbiamo fotografato ci fa ben sperare per una più stretta collaborazione, dettata anche dalla necessità nel caso albanese, vista la predominanza del settore bancario in tutto il mercato e il movimento delle banche verso i nuovi mercati regolamentati. Inutile non accorgersene del fatto che le banche, specialmente le filiali d'importanti gruppi europei ${ }^{4}$, saranno gli attori principali anche nel nuovo mercato dei titoli di credito. Questa dimensione trasversale di sviluppo e di crescita imprenditoriale deve per forza accompagnarsi anche con una vigilanza trasversale verso i mercati, di concerto tra le due autorità.

\section{Il ruolo dell'Autorità nell'integrazione europea e la sua collaborazione con autorità estere di vigilanza}

\footnotetext{
${ }^{1}$ Art. 15, pt. 2, n.10 della I. 9572 del 03.07.2006 "Sull'Autorità di Vigilanza Finanziaria"

2 Vedi supra p. 72

${ }^{3}$ Art. 17 della I. 9572 del 03.07.2006 "Sull'Autorità di Vigilanza Finanziaria"

${ }_{4}$ Portiamo, come esempio, una delle realtà più importanti italiane in Europa come la Intesa-San Paolo che ha deciso di entrare con le sue filiali nel mercato albanese incorporando la Banca Italo-Albanese, gia di sua proprietà.
} 
Dobbiamo dire che l'Autorità di Vigilanza Finanziaria ha anche una dimensione internazionale. Accanto alla sua funzione di vigilanza interna essa ha anche il compito di avvicinarsi alle pratiche delle istituzioni estere di vigilanza, specialmente quelle che operano in condizioni simili al mercato albanese ${ }^{1}$, e apprendere dalle loro esperienze.

Un altro compito della nuova Autorità consiste anche nel diventare parte delle associazioni internazionali delle autorità di vigilanza dei mercati. Dalla sua costituzione fino adesso l'Autorità è entrata a far parte dell'associazione delle autorità che agiscono nel campo delle assicurazioni (International Association of Insurance Supervisors), nel campo dei titoli di credito (International Organisation of Securities Commissions) e nel campo delle pensioni private (International Organisation of Pension Supervisors).

Attualmente, l'Autorità ha sottoscritto un memorandum d'intesa con l'International Organisation of Securities Commissions $^{2}$. L'Autorità, quindi, si è impegnata a conformarsi ai principi della IOSCO è a colmare le lacune legislative e il quadro regolamentare della sua attività.

La collaborazione con le altre autorità nazionali e con gli organismi internazionali, governati e non, è una necessita in questi tempi dove i mercati si stano integrando sempre di più e il capitale investito è di differenti provenienze. In Albania, come abbiamo detto, in questi anni è entrato in maniera preponderante nei mercati il capitale estero (Minxhozi, 2009, p. 41), sia di partecipazione, che di controllo. Fatto questo che ha reso necessario la collaborazione con i paesi d'origine di questi investitori per avere le informazioni necessarie al fine di poter esercitare una vigilanza effettiva ${ }^{3}$.

La ragione della collaborazione non si ferma qua. A loro volta anche le autorità di vigilanza estere hanno un serio interesse a collaborare con un'AVF forte e che vigila il mercato in maniera efficiente. Un interesse che è vivo perché cosi sono certi che i soggetti da loro vigilati, lo saranno anche nei mercati albanesi, non si possono avventurare in situazioni pericolose. Cosi, vista la "giovane età" dell'Autorità, i partner esteri lo assistono nello svolgimento dei propri compiti e le prestano assistenza tecnica.

Negli adempimenti dell'Autorità verso un'affermazione e riconoscimento nel campo internazionale si possono menzionare gli stretti rapporti tra l'AVF e vari organismi internazionali che sono coronati in progetti di leggi o regolamenti compilati in collaborazione, nella formazione del personale, etc. Più in dettaglio possiamo menzionare la collaborazione con la Banca Mondiale e il Fondo Monetario Internazionale nei progetti di legge come "Sui Fondi delle Pensioni Volontarie" e vari regolamenti nel campo della metodologia di vigilanza e per il rafforzamento del quadro regolamentare. Esiste anche una collaborazione con organismi internazionali governativi, come United States Agency for International Development (USAID), o non-profit come il Financial Services Volunteer Corps (FSVC). Collaborazione che si attua sempre preminentemente nel campo del legal drafting.

Un altro partner dell'AVF è stato anche la Banca Europea per Ricostruzione e Sviluppo (BERS) con il quale, a parte l'assistenza nel preparare i disegni di legge ${ }^{4}$, si sta portando avanti un progetto importante sulla costruzione di una piattaforma per la gestione delle informazioni periodiche inviate dai soggetti vigilati (AMF, 2008, p. 37).

Un'osservazione a parte merita la collaborazione con le istituzioni comunitarie. Come ogni altra istituzione albanese anche l'Autorità di Vigilanza Finanziaria è coinvolta nel processo d'integrazione europea del paese. Nell'ambito del Piano Nazionale per l'attuazione dell'Accordo sulla Stabilizzazione e Associazione l'Autorità ha collaborato intensamente con il Ministero dell'Integrazione per adempiere il suo compito, che è quello di armonizzare la legislazione albanese a quella comunitaria anche nel campo dei mercati regolamentati. Tutti i cambiamenti nell'ordinamento intrapresi nel 2008 sono lo specchio di un assiduo lavoro per il recepimento delle direttive comunitarie nel campo delle assicurazioni, pensioni ${ }^{5}$ e titoli di credito(AMF, 2008, p. 35-36). I nuovi progetti di legge di cui abbiamo parlato vanno in questa direzione.

\footnotetext{
1 Per esempio, esiste un intesa per lo scambio di informazioni e di esperienza con l'autorità slovena di vigilanza. (AMF, 2008, p. 37)

2 Final update of 34-th Annual Conference of the International Organization of Securities Commissions, Tel Aviv, 11 giugno 2009.

Documento reperito nel sito della IOSCO: www.iosco.org

3 Importante menzionare la stretta collaborazione con l'autorità austriaca, visto anche gli interessi del capitale austriaco nel mercato albanese delle banche e delle assicurazioni (AMF, 2008, p. 36-37)

4 Un progetto di legge importante che si presenterà in parlamento durante la nuova legislatura nel 2009 è "Sulle obbligazioni delle società per azioni e delle autonomie locali". In: www.amf.gov.al

${ }^{5}$ Manca tuttora una normativa in linea con la direttiva 2003/41/EC "On the activities and supervisions of institutions for occupational retirement provision" per quanto riguarda la normativa delle pensioni private. La legge di riferimento rimane, anche se ammendato nel
} 
Già la legge sull'Autorità è espressione della normativa comunitaria sui mercati regolamentati, sulle assicurazioni e sulle pensioni. La più importante direttiva, senz'altro è la MiFID per i mercati di capitale. La dir. 2004/44/EC (dir. MiFID) sostituisce la dir.92/22/EEC che precedentemente disponeva sulla materia dei mercati finanziari. Essa fa parte di un più ampio disegno di riforma che è il Piano d'azione degli strumenti finanziari (Financial Services Action Plan). Questo piano d'azioni è composto da 42 direttive, tra le quali senz'altro la direttiva MiFID è la più importante. Lo scopo della Commissione Europea è che tramite queste direttive si possa creare un mercato unico europeo che possa rivaleggiare con il mercato statunitense. In quest'ottica, anche l'Albania sta accogliendo la nuova normativa europea e continuerà a lavorare in questa direzione.

\section{I soggetti sottoposti alla vigilanza dell'Autorità di Vigilanza Finanziaria}

In Albania da poco si è costituita una nuova autorità per la vigilanza dei mercati finanziari, delle pensioni e delle assicurazioni. La parte dei mercati dei titoli di credito di nostro interesse, quello finanziario, è vigilata dall'AVF tramite un sistema d'informazione. II mercato primario dei titoli di stato è gestito direttamente dalla Banca D'Albania che colloca i titoli nel mercato tramite aste pubbliche. Questi costituiscono la maggior parte dei titoli negoziabili nei mercati secondari. In Albania i mercati secondari sono pochi. Possiamo menzionare solo la Borsa di Tirana s.p.a, il mercato interbancario dei titoli di Stato e il mercato al dettaglio dei titoli di Stato (monitorate tramite il GSRM ${ }^{1}$ ). Le operazioni si svolgono da intermediari autorizzati dall'Autorità di Vigilanza Finanziaria. II GSRM è un sistema informativo ideato dall'AVF con lo scopo di dare una chiara informazione agli investitori sulle transazioni nel mercato secondario. Ogni operatore ha l'obbligo di inviare alla GSRM l'informazione sulle operazioni giornaliere effettuate ${ }^{2}$ ma tali trasmissioni in questo sistema hanno solo carattere informativo è non obbligano l'operatore a vendere a tale prezzo. Gli ultimi dati raccolti sul GSRM mostrano che la geografia delle transazioni non è cambiata molto dal 2008 al 2009. La compravendita nel mercato primario che nel 2008 costituiva il 33,93\% delle transazioni nel 2009 costituisce solo il 29,20\% ma con una crescita delle transazioni effettuate da persone giuridiche. Le operazioni effettuate dai portafogli degli intermediari finanziari invece hanno subito una diminuzione (dal 39,52\% nel 2008 al 34\% nel 2009) ma comunque anche in queste operazioni si conferma il trend di crescita del numero di operazioni effettuate da persone giuridiche. Basso è il numero delle transazioni con i titoli di credito come collaterali (garanzia). Queste transazioni erano e rimangono solo il 0,50\% (AMF, 2009, p. 1). Altri dati che l'Autorità ci offre per il biennio 2008-2009 sono abbastanza chiari per quando riguarda la percentuale degli intermediari coinvolti nelle transazioni. Le vendite dai portafogli degli intermediari finanziari sono state effettuate per il 96,32\% dalla banca Raiffaisen-RZB mentre solo il 3,32\% dalla Intesa-San Paolo. I titoli comprati prima della maturazione della scadenza passano da più banche, come RZB, Intesa-San Paolo, Banca Credins e BKT ma Raiffaisen è la banca che opera più transazioni (AMF, 2009, p. 5). Sono le divisioni finanziarie delle istituzioni di credito quelle che svolgono la maggior parte delle transazioni sui titoli di stato, $\mathrm{i}$ quali sono gli unici titoli nel mercato. Le banche stesse preferiscono gli immobili o terreni come garanzia per i crediti erogati e marginalmente titoli di credito.

\section{Conclusioni}

A prescindere dallo sviluppo del mercato dei titoli di stato i problemi dei mercati dei capitali rimangono. Durante il 2012 non ce stato nulla di nuovo nell'attività della Borsa di Tirana s.p.a nonostante i tentativi di avvicinare le aziende a quotarsi. L'inattività delle aziende è dovuta da fattori come la natura delle imprese, la gestione prevalentemente familiare, crisi, la mentalità nel paese (AMF, 2008, p. 48-49) ma comunque rimane vivo l'interesse dei soggetti esteri verso la Borsa. Tale fatto è incoraggiante e dipende dalle privatizzazioni in atto di imprese statali in settori strategici rendendo appetibili i pacchetti azionari di controllo. Nonostante il lavoro fatto per lanciare le sedi regolamentate in Albania, continua la compravendita delle azioni nel mercato non regolamentato o in quello informale avendo come conseguenza l'aumento dei costi della transazione, l'incertezza sulla proprietà delle azioni e la funzione di barriera all'entrata per investitori esteri che sono abituati a diverse "regole del gioco". Dall'attività del Centro Registrazione delle Azioni notiamo che il numero delle transazioni aventi come oggetto le azioni delle società statali privatizzate si è abbassato di anno in anno perché dopo il

2006, la I. 7943 dello 01.06.1995 "Per pensionet suplementare dhe istitutet private te pensioneve". Come abbiamo detto, ci si aspetta una veloce approvazione del progetto di legge "Sui fondi delle pensioni private", progetto che recepisce le direttive 2003/41/EC, 85/611/EEC e 2004/89/EEC.

1 Government securities retail market.

2 Reg. 8, del 28.09.2005 "Mbi tregun me pakice te bonove te thesarit ne RSH" 
consolidamento dei asseti proprietari non esiste un interesse agli altri pacchetti azionari delle compagnie(AMF, 2008, p. 49). I dati offerti ci dicono che nel 2008 presso la CRA si sono registrate 494 transazioni, quasi il doppio di quelle registrate nel 2007 (AMF, 2008, p. 50).

Speriamo che in Albania sia dato un input al mercato e che l'imminente integrazione europea possa essere uno dei principali fattori. Comunque, esiste la possibilità che il mercato albanese per le sue dimensioni sia un annesso marginale al mercato europeo. Questa convinzione si forza sempre di più dalla nazionalità dei gruppi bancari e assicurativi che sono attivi nel mercato. Dopo la nuova privatizzazione degli anni 2005-06 le più importanti società ex-statali bancarie e assicurative sono finite in mano ai maggiori gruppi europei e nonostante sia considerato fattore di crescita indiscutibile non deve fungere da limite per altri che vogliono accedere al mercato.

\section{Bibliografia}

[1] A.SMiтH, Wealth of Nations, libro I, cap. X, parte II, p.155

[2] AMF, (2007) Raporti i mbikqyrjes 2007, Tirane

[3] AMF. (2008). Raporti vjetor 2008, Tirane

[4] AMF. (2009) Statistics-Government securities retail market January-June, Tirane,

[5] B.MINXHOZI, (2009) Tendenca ne industrine e sigurimeve ne Shqiperi in Economicus, Tirane: UET Press, nr.2

[6] F.VELLA (2008) Le autorità di vigilanza: non è solo questione delle architetture in Imprese e investitori: Crescita, tutele, interessi, a cura di G.D.Mosco, F.VelLA, Milano: Giuffrè Ed.

[7] Gazeta Standard, Tregu finanziar: AMF nuk mund te shmange detyren e gjallerimit, 30.06.2008

[8] IAIS (2002) Principles on capital adequacy and solvency. nr.5 